\title{
SORTIA 2.0:Um jogo de ordenação para o ensino de Estrutura de Dados
}

\section{Alternative Title: SORTIA 2.0: A sorting game for data structure teaching}

\author{
Paulo Eduardo Battistella \\ Grupo de Qualidade de Software \\ (GQS) Universidade Federal de Santa (G \\ Catarina (UFSC) \\ paulo@incod.ufsc.br \\ Giani Petri \\ Grupo de Qualidade de Software \\ (GQS) Universidade Federal de Santa \\ Catarina (UFSC) \\ giani.petri@posgrad.ufsc.br
}

Aldo von Wangenheim

Instituto Nacional para Convergência Digital (INCoD)

- Universidade Federal de Santa Catarina (UFSC)

awangenh@incod.ufsc.br

\author{
Christiane Gresse von \\ Wangenheim \\ Grupo de Qualidade de Software \\ (GQS) Universidade Federal de Santa \\ Catarina (UFSC) \\ gresse@inf.ufsc.br
}

\author{
Jean Everson Martina \\ Laboratório de Segurança em Computação \\ (LabSec)- Universidade Federal de Santa Catarina \\ (UFSC) \\ everson@inf.ufsc.br
}

\begin{abstract}
RESUMO
O ensino de algoritmos de ordenação nas disciplinas de estrutura de dados, dos cursos da área de Computação, tipicamente é realizado por meio de aula-expositiva e implementação de algoritmos pelos alunos. Com objetivo de apoiar o ensino de algoritmos, o artigo apresenta o jogo SORTIA 2.0, que visa ensinar o algoritmo de ordenação Heapsort através da simulação da sua execução. Ele é um jogo online, single player e gratuito. Para a avaliação sistemática do jogo foi realizado um estudo de caso utilizando o modelo MEEGA, tendo como amostra 25 alunos da disciplina de estrutura de dados do curso de Ciência da Computação da Universidade Federal de Santa Catarina (UFSC). Os resultados da avaliação evidenciam que os alunos se sentiram satisfeitos, confiantes, imersos e se divertiram com o jogo. Mas principalmente consideraram que ele realmente contribui para o aprendizado na disciplina e também profissionalmente.
\end{abstract}

\section{Palavras-Chave}

Aprendizagem Baseada e Jogos; Design de Jogos; Algoritmo de Ordenação; Educação. player and free. To evaluate the game we used the model for assessment educational game MEEGA. The evaluation involved 25 students of the discipline of data structure of Computer Science course, of the Federal University of Santa Catarina (UFSC). The results of this first evaluation show that students felt satisfied, confident, immersed and have fun with the game, but principally consider that it really contributes to learning the discipline and also professionally.

\section{Categories and Subject Descriptors}

\section{K.3.1[Computer Uses In Education]}

\section{General Terms}

Design, Experimentation, Human Factors.

\section{Keywords}

Game Based Learning; Game Design; Sort Algorithm; Education.

\section{INTRODUÇÃO}

Os conteúdos nas disciplinas dos cursos da área de Computação são extensos e normalmente apresentados por meio de aulasexpositivas $[1 ; 2]$. Este tipo de estratégia instrucional é adequada para apresentar conceitos abstratos e informações factuais para um grande grupo de alunos. Porém, não é o mais adequado quando se pretende adquirir objetivos de aprendizagem em níveis mais elevados, que promovam a aplicação do conhecimento em situações práticas [3]. Além disso, aulas-expositivas são centradas no professor, nas quais os alunos participam como receptores, o que muitas vezes se torna cansativo [3].

Objetivando tornar os conteúdos de Computação mais atrativos aos alunos, utiliza-se atualmente a Aprendizagem Baseada em Jogos (Game-based Learning - GBL) [4]. A GBL trata de 
aplicações de jogos nas quais o resultado é a aprendizagem de um determinado conteúdo. Geralmente, estes jogos são concebidos de modo a equilibrar o conteúdo com a jogabilidade [4].

Nesse contexto, um jogo pode ser definido como "qualquer competição (jogo) entre os adversários (jogadores) que operam sob restrições (regras) para um objetivo (vitória ou lucro)" [4].E jogos educacionais, também chamados de jogos sérios (serious games) são especificamente projetados para ensinar as pessoas acerca de um determinado assunto, expandir conceitos, reforçar o desenvolvimento, ou auxiliá-los, exercitando ou aprendendo uma habilidade ou buscando uma mudança de atitude enquanto jogam [4,5].

Atualmente, existem diversas iniciativas para tornar os cursos de Computação mais atrativos. Por exemplo, a complementação do ensino de programação apresentada por Digiampietri et al. (2012) [6], a análise dos cursos de Ciência de Computação buscando o aprimoramento curricular em Gerab, Gerab e Bueno (2014) [7] e análise curricular dos cursos de Sistemas de Informação em Albuquerque et al. (2014) [8].

Entretanto, a utilização de jogos torna o aprendizado mais divertido e atrativo, sendo possível identificar diversos jogos (digitais ou não-digitais) que buscam contribuir com o ensino de Computação. São exemplos destes jogos: Simulação para ensinar SCRUM [9], Problems and Programmers [10] e X-MED [11] para ensinar engenharia de software, No Bug's Snack Bar [12], C-Jump [13], Saving Princess Sera [14] para ensinar programação e AntiPhishing Phil [15] na área de segurança.

Em áreas eminentemente técnicas, como Estruturas de Dados, a utilização de jogos ainda é muito tímida. Existem alguns jogos que são voltados apenas para testar o conhecimento de caráter enciclopédico, sem gerar vivências práticas do conteúdo [16]. Por outro lado, existem trabalhos voltados a jogos baseados em pequenas maratonas de programação [17], mas que não focam no ensino de algoritmos de ordenação.

Considerando que o nível de envolvimento do aluno durante o processo de aprendizado tem papel chave na qualidade e profundidade do aprendizado atingido $[18,19,20]$, este trabalho objetiva apresentar um jogo de ordenação para os alunos da disciplina de estruturas de dados simularem a ordenação. Assim, poderão vivenciar de modo prático o processo de ordenação de um conjunto de números inteiros.

A justificativa para a escolha do algoritmo de ordenação se dá por ser uma temática que vinha sendo abordada de modo bastante abstrato pelos autores. Eram realizadas aulas-expositivas com análise estrutural dos algoritmos. No entanto, ficou evidente para os autores a dificuldade de compreensão conceitual por parte dos alunos. Assim, foi escolhido o algoritmo Heapsort, pois apresenta um grau de dificuldade de aprendizado bastante desafiador, sendo um algoritmo que na experiência dos autores demonstrou ser de aprendizado inicialmente difícil.

O artigo está estruturado nas seguintes seções: (2) método utilizado na pesquisa; (3) trabalhos relacionados; (4) desenvolvimento do jogo; (5) aplicação do jogo em sala de aula; (6) conclusões da pesquisa.

\section{MÉTODO}

O jogo de ordenação possui o nome de SORTIA, tendo a primeira versão publicada no Simpósio Brasileiro de Informática na Educação sob o título SORTIA - Um Jogo para Ensino de
Algoritmo de Ordenação: Estudo de caso na Disciplina de Estrutura de Dados [21].

Os resultados da avaliação do jogo em sua primeira versão, indicaram uma necessidade de melhorar a interface do jogo, além de aumentar o nível de atratividade. A primeira versão foi desenvolvida em HTML5 e JavaScript, utilizando hexágono e quadrados para criar o tabuleiro e os ponteiros (Figura 1).

A primeira versão do jogo foi desenvolvida com base nos modelos ADDIE e ISD, do design instrucional. Por meio do design instrucional é possível criar "experiências de ensino que fazem a aquisição de conhecimentos e habilidades mais eficiente, eficaz e atraente" [24].

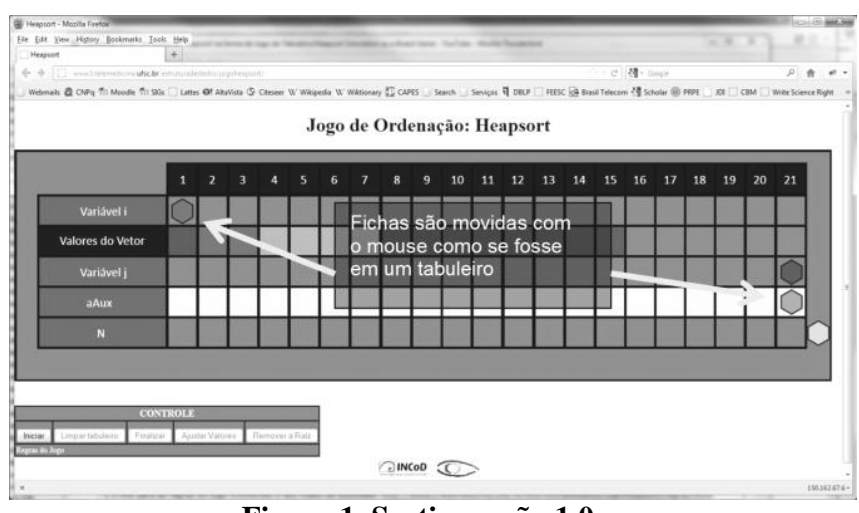

Figura 1. Sortia versão 1.0.

A segunda versão do jogo, apresentada no presente artigo, além do design instrucional, também utiliza o design de jogos $[25,26$, 27, 28]. A partir do design de jogos foi possível produzir elementos do jogo (tabuleiros, ponteiros, botões) mais elaborados, criar regras claras, tornar o layout mais intuitivo e desenvolver a competição entre os alunos por meio de um ranking [25, 26, 27, 28].

Para a avaliação do jogo, foi realizado um estudo de caso utilizando o modelo de avaliação de jogos educacionais MEEGA [29]. O MEEGA avalia a percepção dos alunos após jogarem o jogo sob o ponto de vista da motivação dos alunos (atenção, confiança, relevância e satisfação), experiência do usuário (competência, desafio, divertimento, interação social, imersão) e aprendizagem (contribuição para vida profissional, eficiência na aprendizagem e contribuição no aprendizado na disciplina).

\section{TRABALHOS RELACIONADOS}

Além dos trabalhos apresentados anteriormente, apresenta-se a seguir alguns trabalhos relacionados a pesquisa.

Na pesquisa de Bittar et al. (2015) [30] foi criado e testado um jogo digital e interativo de tabuleiro com perguntas e respostas para aplicação no ensino fundamental.

Na pesquisa de Ferreira et al. (2014) [31] é apresentado o jogo Usabiliti City que busca ensinar a técnica de inspeção de usabilidade por meio de avaliação heurística.

Na pesquisa de Lopes et al. (2013) [32] é apresentado o InspSoft 2.0 que ensina a inspeção de software visando a qualidade dos artefatos de softwares desenvolvidos.

Na pesquisa de Marques, Lopes e Conte (2014) [32] é realizada avaliação utilizando dois métodos de avaliação da experiência dos usuários por meio do jogo uTest. Neste jogo realiza-se simulações de cenários, nas quais o jogador precisa simular testes de software. 
Os trabalhos relacionados evidenciam a utilização de jogos no ensino fundamental e na graduação, sendo tipicamente jogos digitais. Destaca-se que dos jogos encontrados, inclusive com base na revisão sistemática da literatura [32], grande parte são jogos da área de Engenharia de Software. Destaca-se que ainda existem poucos jogos para ensinar algoritmos de ordenação nas disciplinas de Estrutura de Dados, de modo a simular uma execução real do algoritmo. Normalmente os jogos para esta área são teóricos e não permite ao aluno aprender executando os passos reais do algoritmo.

\section{DESENVOVIMENTO DO JOGO}

$\mathrm{O}$ desenvolvimento da nova versão do SORTIA iniciou com a prototipação de baixa fidelidade das novas telas do jogo, tendo como base Design de Jogos [25, 26, 27, 28].

As primeiras telas foram criadas utilizando papel e lápis. $\mathrm{Na}$ Figura 2 é possível verificar que a nova versão está mais clean, menos confusa para o aluno e permite distinguir o tabuleiro dos ponteiros (fichas).

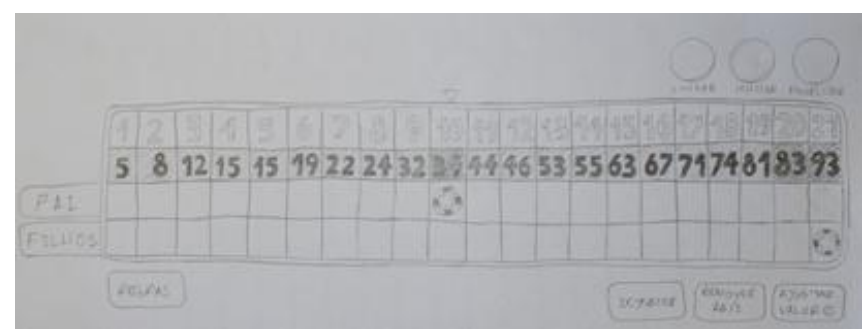

Figura 2. Protótipo de baixa fidelidade.

Para a segunda versão do jogo, foi possível tornar o tabuleiro mais claro, unindo os ponteiros "i" e "aAux" no ponteiro "Pai". O ponteiro "j" mudou para ponteiro "Filho". No jogo foi automatizada a função de controlar o tamanho do vetor de dados, deste modo foi possível retirar a linha "N" apresentada na versão 1.0 (Figura 1).

Os botões do jogo também foram modificados, com objetivo de agrupá-los por funções. Os botões de limpar, iniciar e finalizar foram inseridos no canto direito superior ao tabuleiro. Os botões de controle do algoritmo, como trocar valores e ajustar o tamanho do vetor foram inseridos no canto direito inferior ao tabuleiro. $\mathrm{O}$ botão regra do jogo foi separado dos dois grupos de botões, ficando no canto esquerdo inferior ao tabuleiro.

Após a finalização do primeiro protótipo, foi iniciada a prototipação de alta fidelidade do jogo. As telas foram criadas a partir de uma ferramenta de edição de imagem proprietária. $\mathrm{Na}$ Figura 3, é possível visualizar um exemplo de tela criada para nova versão do jogo.

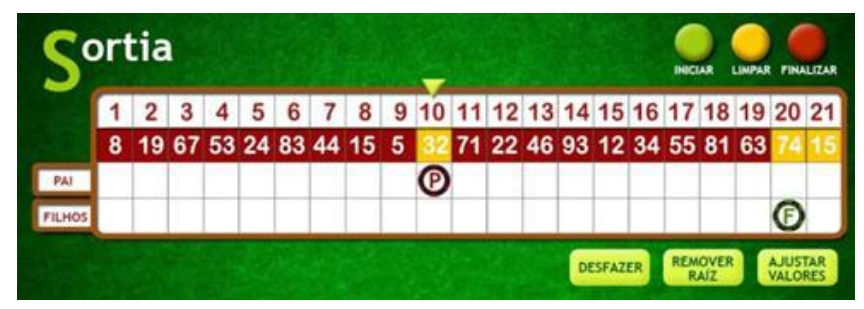

Figura 3. Protótipo de alta fidelidade.

Em comparação com a Figura 1, é possível perceber a diferença de layout do jogo para o protótipo de alta fidelidade. Na Figura 3 a primeira linha do tabuleiro corresponde aos índices do vetor (posições 1 à 21) e a segunda linha aos valores inteiros (de 0 a 100 criados aleatoriamente) que serão ordenados segundo os critérios de ordenação do algoritmo Heapsort.

Após a finalização do protótipo de alta fidelidade, foi iniciado o desenvolvimento do jogo SORTIA 2.0. O jogo foi implementando nas linguagens de programação HTML5, JavaScript e PHP. Na Figura 4 é possível verificar a versão final do jogo, estando disponível gratuitamente no endereço http://incod.ufsc.br/sortia/.

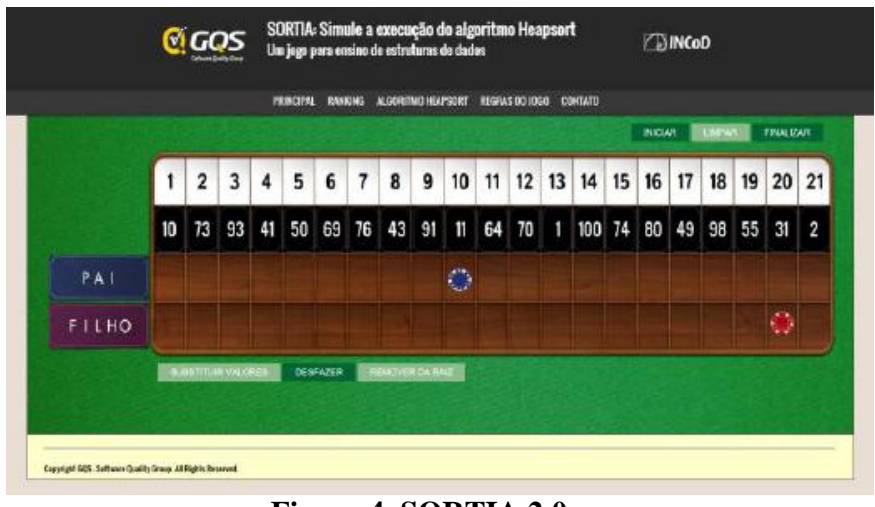

Figura 4. SORTIA 2.0

Algumas modificações foram realizadas da versão protótipo para a versão final do jogo, como a criação de um menu contendo acesso do jogo, ranking dos alunos, explicação sobre o funcionamento do algoritmo, regras do jogo e contato.

Nesta versão, foi mantido os botões iniciar, limpar e finalizar no canto direito superior ao tabuleiro, mas os botões substituir valores, desfazer e reduzir o tamanho do vetor, foram movidos para o lado esquerdo no canto inferior do tabuleiro.

Para jogar, o aluno precisa clicar no botão iniciar, em seguida são criados automaticamente 21 números aleatoriamente de 0 a 100 . Na primeira linha do tabuleiro o aluno poderá visualizar os índices do vetor, sendo que os números a serem ordenados estão visíveis na segunda linha do tabuleiro. O jogador utiliza os ponteiros (fichas) "Pai" e "Filho" para ordenar os dados segundo os passos do Heapsort.

O jogo é single player, é do gênero simulação e desenvolvido para ser jogado online gratuitamente.

\section{APLICAÇÃO DO JOGO}

O jogo foi aplicado na disciplina de Estruturas de Dados no curso de Ciência da Computação da Universidade Federal de Santa Catarina (UFSC). A aplicação ocorreu no segundo semestre de 2015, tendo a participação de 25 alunos. No curso, esta disciplina tem como pré-requisito a disciplina de Programação Orientada a Objetos II, o que evidencia um conhecimento básico de programação dos alunos.

O tempo estimado da aplicação do jogo foi de uma hora. Porém, antes do jogo ser aplicado, o professor da disciplina (um dos autores) apresentou durante uma hora o conteúdo do algoritmo Heapsort.

\subsection{Avaliação}

Para avaliação do jogo foi utilizado o modelo de avaliação de jogos educacionais MEEGA [29]. A avaliação tem por objetivo avaliar o jogo do ponto de vista da motivação, experiência dos 
usuários e aprendizagem. O modelo também permite avaliar os objetivos de aprendizagem.

O gráfico de motivação (Figura 5) evidencia que $80 \%$ dos alunos se sentiram satisfeitos com o jogo, $68 \%$ dos alunos considerando que por meio do esforço pessoal avançaram no jogo. Em relação a confiança, $88 \%$ dos alunos se sentiram confiantes em aprender, e 20 alunos informaram que foi fácil aprender a jogar. Em relação a relevância do jogo, $84 \%$ dos alunos consideraram que o jogo está conectado com outros conhecimentos que já possuíam, 100\% dos alunos admitiram que o jogo funciona do modo como gostam de apreender, e $96 \%$ dos alunos consideraram que o jogo é relevante para seus interesses.

Em relação a atenção, $92 \%$ dos alunos informaram que o design do jogo ajudou a mantê-los atentos no jogo, $60 \%$ dos alunos consideraram que o jogo capturou a sua atenção enquanto jogavam, e $96 \%$ dos alunos consideraram o design do jogo atraente.

Em relação a experiência do usuário, apresentado no gráfico da Figura 6. Observa-se que $88 \%$ dos alunos apresentaram sentimentos positivos de eficiência ao jogar, 92\% dos alunos informaram que conseguiram atingir os objetivos do jogo com suas próprias habilidades. Em relação ao divertimento, $80 \%$ dos alunos pretendem jogar o jogo novamente, $84 \%$ dos recomendariam o jogo para seus colegas, apenas $40 \%$ alunos comentaram que gostariam de continuar jogando o jogo quando foram interrompidos, $92 \%$ dos alunos informaram que se divertiram jogando o jogo.

Em relação ao desafio, $64 \%$ dos alunos informaram que o jogo evolui no ritmo adequado e $76 \%$ dos alunos informaram que o jogo apresenta desafios adequados ao jogador. Em relação a interação social, mesmo sendo um jogo single player o jogo foi avaliado positivamente, tendo $92 \%$ dos alunos informando que o jogo promove a competição entre os jogadores, possivelmente relacionado ao ranking dos jogadores, $80 \%$ dos alunos comentaram que se divertiram jogando com outras pessoas, possivelmente porque estes alunos dividiram o computador com o colega.

Em relação a imersão, apenas $48 \%$ dos alunos informaram que tiveram boa imersão no jogo, $68 \%$ dos alunos informaram que não perceberam o tempo passar enquanto jogavam, e $60 \%$ dos alunos esqueceram das preocupações durante o jogo.

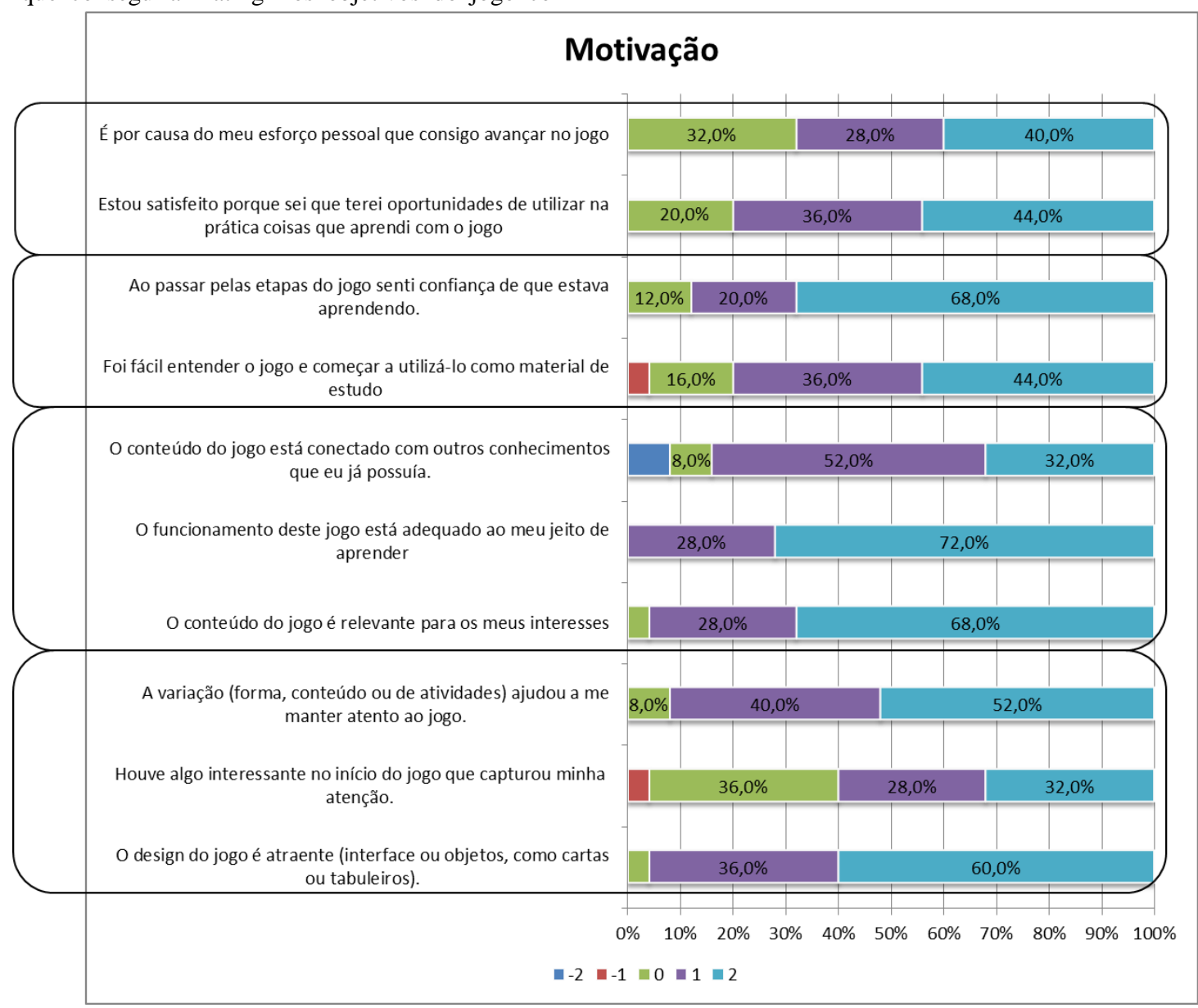

Figura 5. Gráfico de motivação dos alunos. 


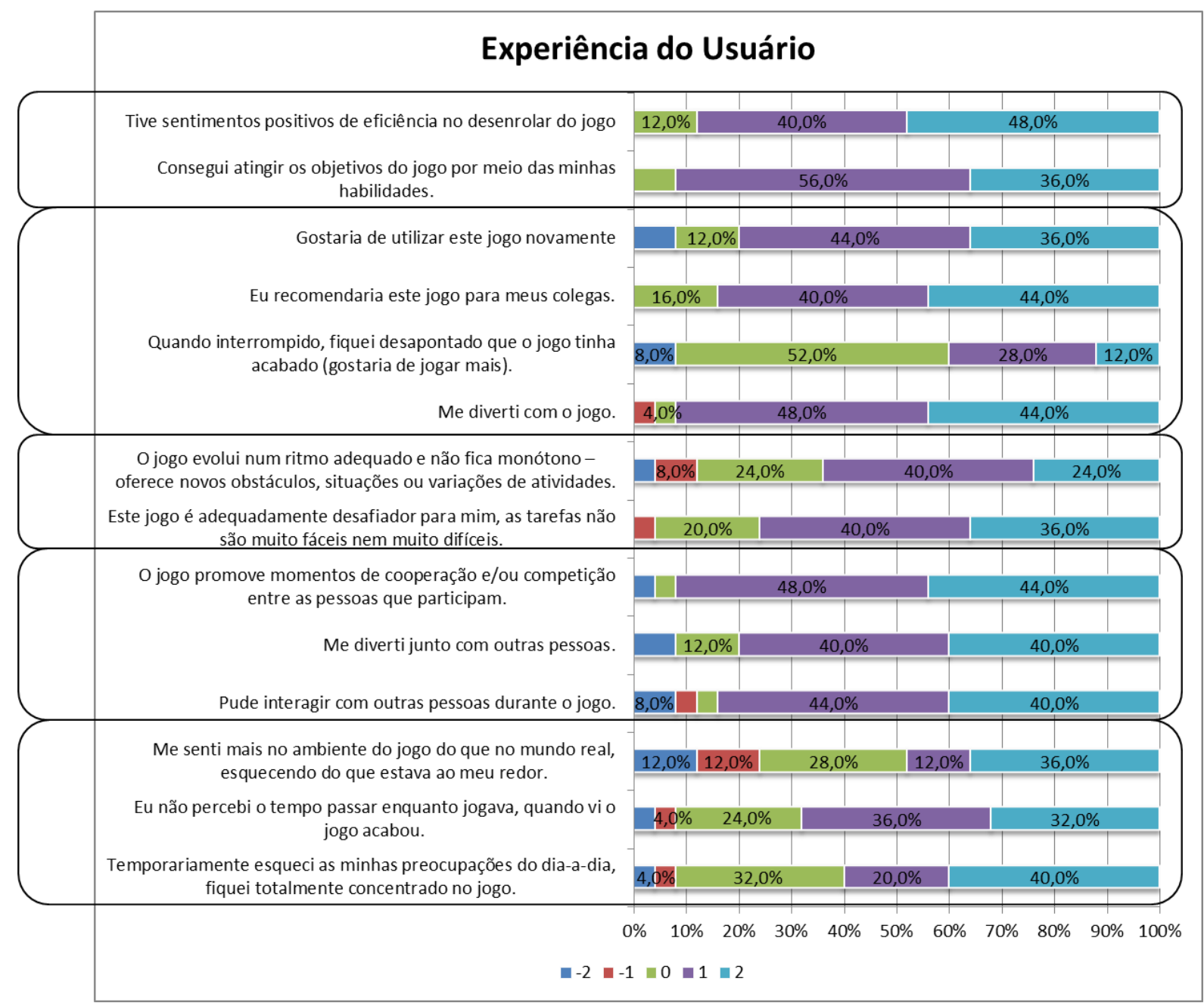

Figura 6. Gráfico de experiência dos usuários.

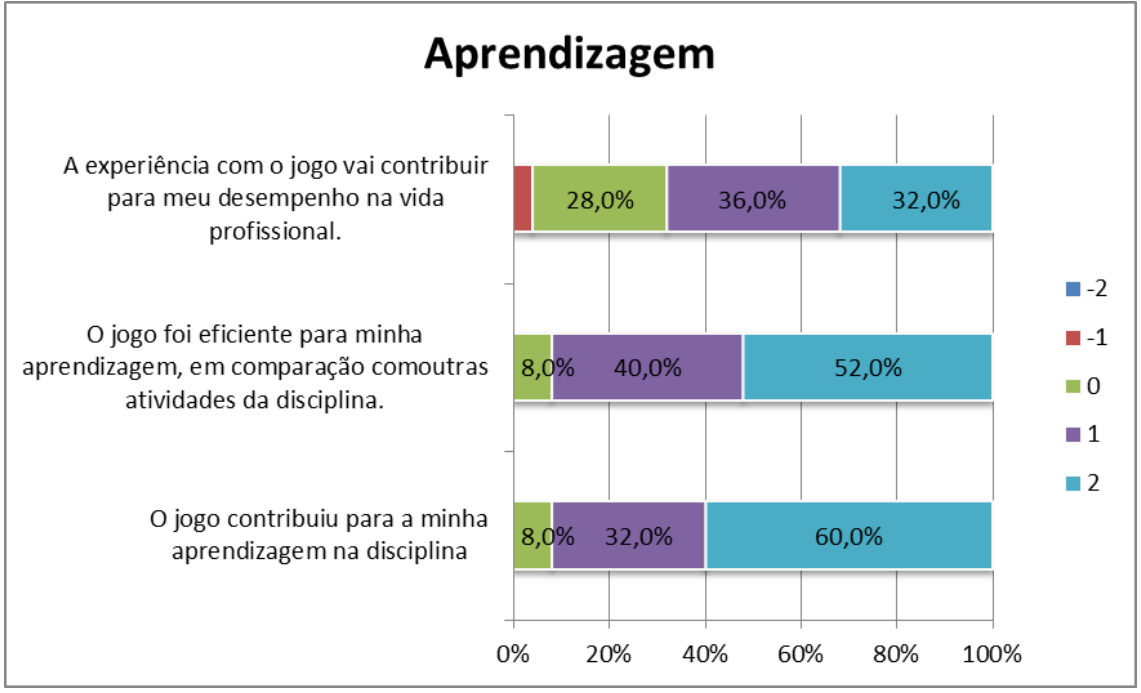

Figura 7. Gráfico da aprendizagem. 


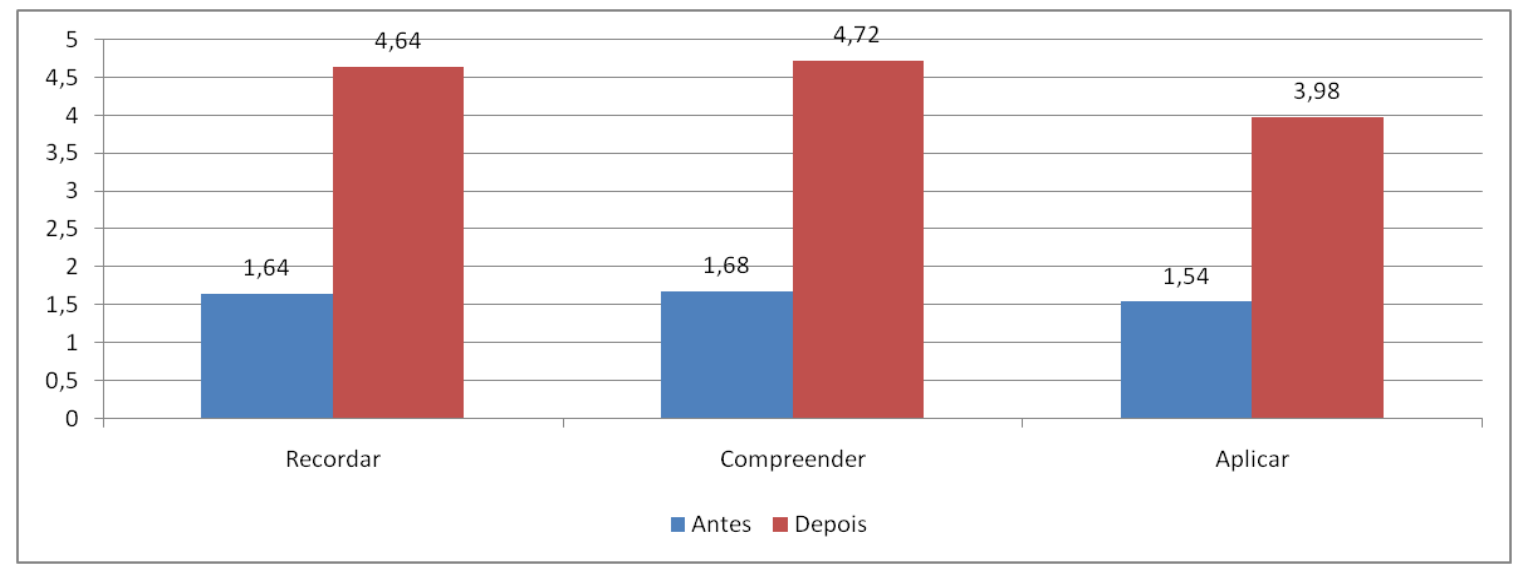

Figura 8. Gráfico dos objetivos de aprendizagem.

O gráfico de aprendizagem (Figura 7) evidencia que 68\% dos alunos consideram que o jogo contribuiu para seu aprendizado profissional, 92\% dos alunos consideraram o jogo apresenta uma forma de aprendizagem mais eficiente que outras atividades da disciplina, e $92 \%$ dos alunos consideraram que o jogo contribuiu para o aprendizado na disciplina. No aspecto da aprendizagem, fica evidente que a grande maioria dos alunos consideraram o jogo importante e relevante para sua aprendizagem.

O gráfico de objetivos de aprendizagem (Figura 8) evidencia que houve um aumento no nível de conhecimento dos alunos após a aplicação do jogo. Em uma escala de 0 a 5, houve um aumento de 3 pontos em relação a recordar o conteúdo após o jogo, houve um aumento de 3,04 pontos em relação a compreensão do conteúdo depois do jogo, e houve um aumento de 2,44 pontos em relação a aplicação do conteúdo. Este gráfico evidencia que os alunos aprofundaram o conhecimento do algoritmo Heapsort, aplicando na prática.

\subsection{Discussão}

Considerando a nova versão do jogo SORTIA, foi possível perceber maior motivação dos alunos em aprender o algoritmo Heapsort. A avaliação teve resultados positivos nos itens satisfação, confiança, relevância e atenção, o que potencializa a motivação para o aluno continuar jogando e aplicando na prática os conceitos do algoritmo Heapsort.

Em relação a experiência dos usuários, os alunos avaliaram bem os itens competência, divertimento, desafio e interação social. Porém, no item imersão, pouco mais da metade dos alunos avaliaram bem o jogo. Possivelmente os alunos avaliaram bem o item interação social porque jogaram em grupos de dois alunos por computador e também porque o jogo apresenta um ranking com os melhores alunos que resolveram a ordenação em menor tempo.

Em relação a aprendizagem, os alunos consideraram que o jogo contribuiu para desenvolvimento profissional. O que evidencia a sua eficiência no ensino em comparação com outras atividades e que contribuiu com o aprendizado na disciplina.

Em relação os objetivos da aprendizagem, os alunos afirmaram que o jogo facilitou a recordação, compreensão e aplicação do conteúdo.

\section{Ameaças a validade da pesquisa}

Em uma pesquisa, é possível considerar como ameaça todos os fatores que influenciam negativamente o resultado da pesquisa. $\mathrm{Na}$ presente pesquisa, considera-se como ameaça o fato da pesquisa ter sido realizada com apenas uma turma. Para minimizar esta ameaça, serão realizadas pesquisas futuras em outras turmas dos cursos de CC e SI. Entretanto, os resultados iniciais evidenciam boa aceitação dos alunos para o jogo.

Outra ameaça a validade é a possibilidade dos alunos atribuírem notas mais altas ao jogo, somente para "agradar o professor" da disciplina. Para minimizar esta ameaça foi informando aos alunos que a avaliação não teria nota e seria anônima.

Os primeiros resultados da aplicação do jogo SORTIA 2.0 evidenciam a importância da utilização de jogos no apoio ao ensino de algoritmos nos cursos de Computação. Entretanto, é necessário realizar outras aplicações do jogo em outras turmas para compreender melhor os efeitos da utilização do jogo na aprendizagem dos alunos em um contexto mais amplo.

\section{CONCLUSÃO}

O Aprendizado Baseado em Jogos é um modo de tornar o ensino de Computação mais atrativo aos alunos. Porém, é necessário verificar se os jogos utilizados apresentam equilíbrio entre conteúdo e a jogabilidade.

Neste sentido, o artigo apresentou uma nova versão do jogo SORTIA. O jogo ensina o algoritmo de ordenação Heapsort, o qual estava sendo ensinado anteriormente por meio de aulas expositivas. No seu desenvolvimento ele utiliza aspectos do Design Instrucional e Design de jogos para equilibrar o conteúdo e a jogabilidade.

A versão 2.0 do jogo foi desenvolvida em HTML5, JavaScript e PHP, possui modo de interação sigle player, é do gênero simulação, acessível via internet e gratuito.

A primeira avaliação do jogo contou com a participação de 25 alunos da disciplina de Estruturas de Dados da universidade, os quais jogaram e avaliaram o jogo. $\mathrm{O}$ resultado da avaliação evidenciou que os alunos se sentiram satisfeitos ao jogar, sentiram confiança no que estavam aprendendo, consideraram o jogo relevante para o ensino do algoritmo, consideraram o design do jogo atrativo, sentiram que o jogo desenvolve competências, consideraram o jogo divertido e desafiador. 
Em relação aprendizagem, fica evidente a boa avaliação dos alunos. Eles consideraram que o jogo contribui para o desempenho profissional, que é uma boa opção de aprendizado em comparação a outras atividades educacionais e que o jogo contribui com o aprendizado na disciplina.

Como passos futuros, o jogo será aplicado em outras disciplinas dos cursos de Computação da universidade e também pretende-se desenvolver uma versão do jogo online do algoritmo Quicksort, o qual é apresentado atualmente por meio de um jogo de tabuleiro manual.

\section{AGRADECIMENTOS}

Este trabalho foi apoiado pelo CNPq (Conselho Nacional de Desenvolvimento Científico e Tecnológico), uma entidade do governo brasileiro focada no desenvolvimento científico e tecnológico e pela CAPES (Coordenação de Aperfeiçoamento de Pessoal de Nível Superior).

\section{REFERÊNCIAS}

[1] ACM/IEEE-CS. 2013. Computer Science Curricula 2013.Estados Unidos da América: ACM e IEEE Computer Society, p.514.

[2] Ministério da Educação. 2012. Diretrizes Curriculares Nacionais para Cursos de Graduação em Computação. Parecer CNE/CNS 136/2012. Disponível em: <http://portal.mec.gov.br>.Acesso em: 13 maio. 2015.

[3] Choi, J. e Hannafin, M. 1995. Situated Cognition and Learning Environments: Roles, Structures and Implications for Design. Educational Technology Research and Development, 43(2), p. 53-69.

[4] Abt, C. C. 2002. Serious Games. University Press of America.

[5] Dempsey, J. V., Lucassen, B. e Rasmussen, K. 1996.The Instructional Gaming Literature: Implications and 99 Sources. TechnicalReport 96-1, College of Education, University of South Alabama.

[6] Digiampietri, Luciano A. et al. 2012. Complementando o Aprendizado em Programação: Experiência no Curso de Sistemas de Informação da UPS. VIII Simpósio Brasileiro de Sistemas de Informação. São Paulo, Brasil.

[7] Gerab, F., Gerab, I. F. da S. e Bueno, I. A. M. 2014. Análise das Interações Curriculares em um Curso de Ciência da Computação: buscando subsídios para aprimoramento curricular. Brasileira de Informática na Educação, 22(1), p. 30-44.

[8] Albuquerque, J. P. et al. 2014.Educação em Sistemas de Informação no Brasil: Uma Análise da Abordagem Curricular em Instituições de Ensino Superior Brasileiras. Revista Brasileira de Informática na Educação, 22(1), p. 7992.

[9] Gestal, P. R. E. e Barros, R. M. de. 2014. Proposta de um Simulador para Auxiliar no Processo de Ensino do SCRUM. X Simpósio Brasileiro de Sistemas de Informação. Londrina, PR, Brasil.

[10] Baker, A., Navaro, E. O. e Hoek, A. v. d. 2003. Problems and Programmers: an educational software engineering card game. Proceedings of the 25th International Conference on Software Engineering, Portland, OR, EUA.

[11] Wangenheim, C. G. v. et al. 2009.Desenvolvimento de um jogo para ensino de medição de software. SBQS - Simpósio Brasileiro de Qualidade de Software, Ouro Preto, Brasil, 2009.

[12] Vahldick, A. et al. 2015.Testando a Diversão em um Jogo Sério para o Aprendizado Introdutório de Programação. $23^{\circ}$ WEI - Workshop sobre Educação em Computação, XXXV Congresso da Sociedade Brasileira da Computação, Recife, Pernambuco, Brasil.

[13] Singh, R. e Singh, J. 2007. Learning Computer Programming Using A Board Game - Case Study On CJump. Master thesis, Multimedia University. Malásia.

[14] Eagle, M. e Barnes, T. 2009. Experimental evaluation of an educational game for improved learning in introductory computing. 40th ACM Tech. Symposium on Computer Science Education, Chattanooga, EUA, p. 321-325.

[15] Sheng, S. et al. 2007. Anti-Phishing Phil: the design and evaluation of a game that teaches people not to fall for phish. 3rd Symp. on Usable Privacy and Security, Pittsburgh, EUA.

[16] Hakulinen, L. 2011. Card games for teaching data structures and algorithms. Proceedings of the 11th Koli Calling International Conference on Computing Education Research. P. 120-121, ACM New York, NY, USA.

[17] Lawrence, R. 2004. Teaching data structures using competitive games. IEEE Transactions on Education Volume 47(4),p. 459-466. IEEE Press Piscataway, NJ, USA.

[18] Dale, E. 1969. Audio-visual methods in teaching. New York: Dryden.3rd ed., Holt, Rinehart \& Winston, New York, p. 108.

[19] Biggs, J. 2003. Teaching for quality learning at university. 2nd ed. Open University Press, Berkshire, UK.

[20] Hamilton, J. e Tee, S. 2010. Smart utilization of tertiary instructional modes. Computers \&Amp, Education, 54(4), May 2010, p. 1036-1053.

[21] Battistella, P. E., Wangenheim, A. von e Wangenheim, C. G. Von. 2012. SORTIA - Um Jogo para Ensino de Algoritmo de Ordenação: Estudo de caso na Disciplina de Estrutura de Dados. $23^{\circ}$ Simpósio Brasileiro de Informática na Educação. Rio de Janeiro, Brasil.

[22] Molenda, M. 2003. In Search of the Elusive ADDIE Model. Performance improvement 42(5),p. 34-37.

[23] Dick, W. e Carey, L. 1996. The systematic design of instruction. 4th ed. New York, NY: Harper Collin.

[24] Merril, M. D. et al. 1996. Reclaiming instructional design. Educational Technology, 36(5), p. 5-7.

[25] Fullerton, T. 2008. Game design workshop: A playcentric approach to creating innovatives games. 3. ed. Burlington: Morgan Kaufmann, p. 470.

[26] Kremes, R. 2009.Level Design: Concept, theory, and practice. A. K. Peters, EUA.

[27] Salen, K. e Zimmerman, E. 2009. Regras do Jogo: fundamentos do design de jogos. Vol. 2, Blucher, São Paulo, BR.

[28] O merinck, M. 2004. Creating the art of the game. New Riders, California, EUA. 
[29] Savi, R.,Wangenheim C. G. von e Borgatto, A. 2011. Um Modelo de Avaliação de Jogos Educacionais na Engenharia de Software. 25th Brazilian Symposium on Software Engineering, São Paulo, BR.

[30] Bittar, T. J. et al. 2015.Uma Abordagem para Gestão de Avaliações de Ensino Baseado em Jogo Interativo de Tabuleiro: O Ludo Educativo Atlantis. XI Simpósio Brasileiro de Sistemas de Informação, Goiânia, GO.

[31] Ferreira, B. M. et al. 2014.UsabiliCity: Um Jogo de Apoio ao Ensino de Propriedades de Usabilidade de Software Através de Analogias. III Congresso Brasileiro de Informática na Educação, XXV Simpósio Brasileiro de Informática na Educação, Dourados, MG.
[32] Lopes, A. ate al. 2013.InspSoft 2.0: Um Jogo para Ensino de Inspeção de Software. XII Simpósio Brasileiro de Qualidade de Software, v. 1, Salvador, Bahia.

[33] Marques, A. B., Lopes, A. e Conte, T. 2014. Aplicação de métodos de avaliação de avaliação da experiência do usuário na utilização de serious game em sala de aula. VII Fórum de Educação em Engenharia de Software, Maceió, AL.

[34] Battistella, P. E. e Wangenheim, C. G. von. 2014. Systematic Literature Review (SLR) of Game-based Learning for Computing. Relatório Técnico, Instituto Nacional para Convergência Digital, INCoD, No 0002/2014-E-GQS. 